\title{
Meeting Report: In Vivo Predictive Dissolution Conference
}

\author{
Johannes Krämer ${ }^{1, *}$ and Erika Stippler ${ }^{2}$ \\ ${ }^{1}$ PHAST GmbH, Kardinal-Wendel-Str. 16, 66424 Homburg, Germany \\ 2 United States Pharmacopeial Convention, Rockville, MD, USA
}

e-mail:ceo-phast@phast.com

\section{ABSTRACT}

T his report details discussions held over three days, August 4-6, 2014, in Ann Arbor, MI, on current research on in vivo predictive dissolution (IPD) testing. Participants hailed from academia, industry, and governmental bodies, and focused their attention on the role of IPD in various drug products. Academia was well represented at this conference with speakers mainly from the University of Michigan, but also from Europe and Japan. Their approach for development of IPD is mainly based on solubility characteristics of the drug substance. A great deal of attention was drawn to the composition and mechanical properties of dissolution media.

Examples presented by speakers from the pharmaceutical industry showed how the findings from academia are implemented in their method development processes.

FDA strongly supports the implementation of new approaches to improve the predictive power of dissolution testing.

For decades, the pharmaceutical scientific community has been debating whether to separate or consolidate the dissolution testing methodologies used in different stages, from development to market supply, of a drug product lifecycle. Moreover, regulatory authorities are consistently challenged to accelerate their decisions on ascertaining bioequivalence (BE) of pharmaceutical drug products (within one product or from product to product). The proof of bioavailability (BA) requires human studies. In vivo predictive dissolution (IPD) testing may reduce the number of BA studies required and could help to speed up marketing authorization and the total time-to-market process for industry.

More than 100 scientists from the United States, Europe, and Japan gathered for three days in Ann Arbor, Michigan, in early August 2014 to develop a white paper that was to serve as an important contribution to the field of in vivo predictive dissolution. U.S. regulatory authorities stimulated the discussions and were ready to consider novel methodologies in their decision-making process.

This report summarizes the scientific presentations given at the conference by experts from pharmacopeias, regulatory authorities, academia, and industry around the globe. This report does not discuss a summary of the white paper, since this document will be published separately (1).

*Corresponding author.

\section{USP-WHERE DISSOLUTION METHODOLOGY IS STANDARDIZED}

Erika Stippler (United States Pharmacopeial Convention, USPC) provided a frequency distribution of the dissolution apparatus stipulated in drug product monographs of the current United States Pharmacopeia (USP).

The majority of the dissolution methods approved by regulatory authorities in the United States were developed using the compendial paddle or basket apparatus. USP dissolution methods and specifications are the same as those approved by the FDA, and USP General Chapter < 711 > Dissolution describes the commonly used apparatus for dissolution testing of oral drug products. In the current USP, there are several chapters that are relevant for dissolution/ drug release testing for various dosage forms. The use of a particular dissolution apparatus depends on the specifics of the drug substance and the dosage form under investigation. A survey of all drug product monographs shows that the most commonly used dissolution apparatus is Apparatus 2, followed by Apparatus 1. Additionally, there are drug product monographs that call for the use of modified compendial apparatus (i.e., alternative basket mesh size, peak vessel, or noncompendial apparatus such as the device termed the reciprocating shaker or tube rotator). For the submission of an abbreviated new drug application (ANDA), the appropriate USP method should be used. If no such method is available, the recently revised USP General Chapter <1092> The Dissolution Procedure: Development and Validation may provide guidance for the development and validation of a suitable method (2).

\section{GREAT INCREASE OF SCIENTIFIC KNOW- HOW AND DEMAND AT FDA \\ FDA Office of Pharmaceutical Science}

Lawrence Yu defined his understanding of the roles of dissolution testing for new and generic drug products to be twofold:

- Quality control tool for the evaluation of batch-to-batch consistency.

- Surrogate for in vivo performance.

The quality standard is a specification established through the clinical performance of the drug product, thus connecting the standard to safety and efficacy; therefore, the quality standard should not be established based on 
the product's variability due to the manufacturing process.

Future roles of dissolution testing should have emphasis on:

- Detecting product changes that affect the clinical performance of the drug product.

- Predicting in vivo performance to reduce the need for human clinical studies.

$\mathrm{Yu}$ reemphasized the fact that classical dissolution testing as a part of quality control (QC) testing may not provide control over drug quality as long as no meaningful approach for optimal dissolution is integrated in the manufacturing process.

\section{FDA Office of Testing and Research}

Are FDA laboratories ready for the new era of dissolution testing?

Since the times of Cox, Furman, and Moore, FDA scientists have been involved in the development of the dissolution apparatus and subsequent improvement of the methods associated with the apparatus. Currently, research in collaboration with other organizations is ongoing at FDA to understand any source of variability in the outcomes of dissolution testing. Any variability in results due to lab artifacts and not the product itself is critical in cases of profile comparison.

One present topic of research focuses on evaluating the hydrodynamics in the compendial apparatus. Computer simulations are used to attempt to improve dissolution methodologies; however, these simulations have yet to address the concern that the outcome of any dissolution methodology often poorly reflects the in vivo performance of the drug product.

More recent FDA research explores whether mechanistic in vitro-in vivo correlation (IVIVC) modeling would be more predictive than the classical approach, starting with numerical deconvolution of the hypothetical dissolution rate. Lucinda Buhse concluded that more than one in vitro dissolution approach may be needed to support the life cycle of a product. This demonstrates that FDA is very open for the development of novel dissolution methods to facilitate, and therefore accelerate, BE decisions.

\section{FDA Office of Generic Drugs}

\section{What about the Office of Generic Drugs at FDA?}

Robert Lionberger leads the implementation of Generic Drug User Fee Amendments (GDUFA) as a regulatory science program, through internal and external research activities (3). In 2012, FDA committed to preparing a yearly list of regulatory science priorities based on input from industry and other stakeholders. For Fiscal Year 2014, FDA's regulatory science priorities for generic drugs are:

- Post-market evaluation of generic drugs.

- Equivalence of complex products such as drug-device combinations, transdermal systems, implants and parenteral microspheres, liposomes, and others.

- Equivalence of locally acting products such as inhalation, topical dermatological, nasal, ophthalmic, and others.
- Therapeutic equivalence evaluation and standards including risk-based equivalence standards for narrow therapeutic index drugs.

- Computational and analytical tools used for developing a modern ANDA review process.

According to Lionberger, the goal is to ensure the therapeutic equivalence of generic drugs. New external collaborations include 20 grants and 8 contracts that total $\$ 17$ million in new regulatory science activity (4). Furthermore, FDA is undergoing reorganization in this field with the development of a new "super office," the Office of Generic Drugs, which will have an Office of Research and Standards with a Division of Therapeutic Performance. The Office of Generic Drugs will also have a Division of Quantitative Methods and Modeling (DQMM), which will provide models to support therapeutic equivalence based on physiologically based pharmacokinetic modeling / absorption models, pharmacodynamics, pharmacometrics (disease computational modeling), and multivariate analytical methods. Lionberger concluded with the statement that IPD requires modeling and simulation, but that modeling and simulation data are rarely submitted in an ANDA. He invited applicants to undertake confidencebuilding steps that include case studies or pilots of how IPD/modeling and simulation can be used in ANDA product development and be effectively interpreted by FDA during the review process.

\section{FDA Center of Veterinary Medicine}

Is in vivo predictive dissolution testing also for veterinary drugs?

The focus of this conference was on IPD for drug products for human use. With regard to the intra- and inter-individual variability of the gastro-intestinal (Gl) physiology, prediction is considered possible at a lower degree of precision. However, precise results are needed for the comparison of different manufacturing variations of one product. This would be meaningful for a new drug application (NDA) or for scale-up and post-approval change submissions to ascertain BE of a lot compared with the lot used for clinical testing of safety and efficacy. As an extension, regulators hope to apply the results of IPD for the comparison of essentially similar products, again to prove $\mathrm{BE}$ for the generic and the pioneer drug product.

Marilyn Martinez provided a perspective on drugs for animal use. In conclusion, the same rules would apply as for human drugs, except that prediction based on IPD is much more challenging due to the complexity and variability of the physiology of animals, such as periodic retrograde peristalsis found in ruminants. Additionally, the size and technology (i.e., buoyant or gastroretentive dosage forms) of animal drugs may require alternative in vitro approaches.

\section{ACADEMIC RESEARCH IN THE UNITED STATES University of Michigan}

Gordon Amidon focused on the fact that IPD is mainly used in product development, though for the purpose of 
market supply (i.e., for $\mathrm{QC}$ testing), other methodologies may be favorable. This decision on methodologies resides within the development history of a product. Still, delegates from regulatory authorities would welcome dissolution methods that would allow them to ascertain the BE of generic products. This implies that methods would be suitable for more than one product to predict the in vivo performance. Currently, laboratories produce in vitro results with lower variability when compared with in vivo human data. The outcome of human studies may depend on a variety of factors as Amidon explained with his study on viscous fluids administered concomitantly with a multiple-unit dosage form (5). The variability of in vivo data also has an impact on the reliability of an IVIVC. Existing mechanistic models are based, to a great extent, on the physicochemical properties of the active pharmaceutical ingredient (API). Predictive dissolution testing should elaborate the impact of the dosage form.

In addition, excipients may play an important role in drug absorption in vivo. To explain these differences between the engineered systems and the human physiology, Gordon Amidon referred to Gregory Amidon's presentation on bicarbonate in vivo buffering systems. For modeling the Gl-transit rate, the proximal section in particular is of greatest importance. Gastric emptying may underlie significant alterations due to the caloric content, volume, and temperature of its content. Amidon recommended the use of subclasses of the Biopharmaceutics Classification System (BCS) as a decision tree starting point (6).

Proposed Relevant IPD Based on the BCS Subclassification (Adapted from ref 6. Copyright 2014 Elsevier).

\begin{tabular}{|l|c|c|c|c|}
\hline \multicolumn{1}{|c|}{ BCS Class } & $\begin{array}{c}\text { Solubility } \\
\text { at } \mathbf{~} \mathbf{H} \text { 2 }\end{array}$ & $\begin{array}{c}\text { Solubility } \\
\text { at pH 6.5 }\end{array}$ & Permeability & Medium \\
\hline I & High & High & High & PIB \\
\hline Ila & Low & High & High & 15 and 30 min in PGB then PIB \\
\hline IIb & High & Low & High & 15 or 30 min in PGB then PIB \\
\hline Ilc & Low & Low & High & $\begin{array}{c}\text { Dissolution 15 and 30 min in PGB } \\
\text { then PIB + surfactant to match in } \\
\text { vivo solubilization }\end{array}$ \\
\hline III & High & High & Low & Same as I \\
\hline IVa & Low & High & Low & Same as Ila \\
\hline IVb & High & Low & Low & Same as Ilb \\
\hline IVc & Low & Low & Low & Same as Ilc \\
\hline
\end{tabular}

PGB: physiological gastric buffer (e.g., $0.01 \mathrm{~N} \mathrm{HCl}$ )

PIB: physiological intestinal buffer (e.g., bicarbonate buffer $\mathrm{pH} 6.5$, buffer concentration 5-15 mM)

Amidon pointed out that in vitro and in vivo volumes are different in cases when the in vivo turnover rate is ignored. Moreover, the emptying kinetics from the stomach (i.e., zero-order vs first-order kinetics) may have an impact on the maximum concentration and other parameters describing the rate of BA. On his wish list to achieve better prediction, Amidon commented that in addition to the volume and the composition of media, the in vivo hydrodynamics should also be considered in the design of any in vitro apparatus.

In cases of drug products with systemic action where the active moiety is absorbed and becomes available in the systemic circulation, the analysis of blood concentration profiles allows the deconvolution of hypothetical in vivo dissolution rates. This is the most common procedure for extended-release (ER) dosage forms, whereas in the case of immediate-release (IR) dosage forms, it is limited to those products for which dissolution is the rate-limiting step. For all other dosage forms and more specifically for locally acting oral drugs with practically no absorption, only the in vivo measurements in the Gl tract provide information about the in vivo dissolution rate.

One way of determining the in vivo dissolution rate is intubation of subjects. Models have been previously introduced in biopharmacy by Lennernäs and others (7), where segments of the gut may be isolated by inflated balloons to control influx and efflux masses over time. Duxin Sun (University of Michigan) presented his results of studies using this and other related techniques.

Based on the BCS, further subclassification for dissolution and permeation characteristics were undertaken for some drug substances and products in vivo. This was used as a starting point for predicting potential in vivo rate-limiting steps. With the identification of the ratelimiting step, the development of the in vivo predictive dissolution method may be started. Biphasic systems with two different media and adjustable influx and efflux rates were advantageous to the "single-compartment" USP Apparatus 1 and 2 (8). One of Deanna Mudie's (University of Michigan) conclusions was that a mechanistic model was successfully developed for BCS Class Ila and IVa drug substances, and that more accurate IPD models can be achieved through better knowledge of in vivo transport mechanisms. Still, all mathematical transport models should be "validated" with additional in vivo testing.

The dissolution of the weak acid drugs ibuprofen, ketoprofen, indomethacin, and the weak base haloperidol were studied by Gregory Amidon's group at the University of Michigan. The solubility of these drugs in bicarbonate and phosphate buffer was investigated using a rotating disk dissolution device. These types of dissolution experiments have to be considered as preliminary studies, because they were conducted with the formulation free drug substance. The mass flux could be predicted for the in vitro dissolution rate implementing a combined diffusion and chemical reaction model, adjustable to different hydration rates based on a modified stagnant layer theory. This is valid for buffers that undergo instantaneous reversible chemical reactions, such as phosphate buffers. As compared with the bicarbonate buffers, phosphate buffers provide substantially greater buffering capacity. This was shown with results obtained with a pure drug substance and the rotating disk method, where concentrations of phosphate buffer between 5 and $30 \mathrm{mM}$ seem to better reflect the physiological conditions. The equivalent phosphate buffer 
concentration may vary depending on the drug substance properties (acid, base, and $\mathrm{p} K_{\mathrm{a}}$ ).

Cocrystals are crystals that contain two or more different molecular components, are solid at room temperature, and are structured in a homogenous (single) crystalline phase with well-defined stoichiometries. They often rely on hydrogen-bond assemblies between neutral molecules of API and other components. The use of cocrystals is one way to overcome the poor solubility of certain drugs substances. Nair Rodriguez-Hornedo (University of Michigan) presented data on a typical hydrophobic drug with a characteristic solubility profile in the presence of surfactants. Only above the critical micelle concentration $(\mathrm{CMC})$ was the proportionality of solubility and surfactant concentration found. However, in the case of cocrystals, the solubility is usually higher below the CMC, whereas above CMC no proportionality was found. Instead, a square-root-versus-time functionality could be established. The solubilizing effect is initially superior to the unbound drug. As soon as the critical stabilization concentration is reached, cocrystals no longer amplify solubility. Their solubility behavior is also dependent on the type of surfactant selected. Cocrystals can exhibit transition points, which are a function of solution additives, $\mathrm{pH}$, and temperature.

\section{U.S. Academia Outside Michigan}

The prediction of relevant in vivo dissolution processes may be undertaken by accurate description of the in vivo motility state of the Gl tract, and the in vivo hydrodynamics in particular, contrasting the fasted and fed state. James Brasseur (Pennsylvania State University) presented a mathematical framework for the prediction of diffusion-dominated dissolution from polydisperse, spherical drug particles. This stepwise procedure found in the framework starts with particles homogeneously distributed in a hypothetical container with impermeable walls. According to Brasseur's investigations, the differences between the geometries of the in vivo and the in vitro sites of dissolution are not critical, though other aspects, such as the confinement in the human intestine, are of greater relevance. In the next step, the physics of the model are then reduced based on experimental findings. Subsequently, the dissolution procedure is then connected to total concentration in this container, the initial particle size distribution, and the saturation concentration. Brasseur's models are based on the assumption that in the human Gl tract, two motility patterns prevail, the "propagation" caused by peristalsis with a more circular pattern and the "standing" due to segmentation characterized by collapsing and reopening movements. In the human gut, dissolution of solids is mostly diffusion dominated and driven by the relationship of particle size to diffusion layer thickness, expressed as the so-called "Sherwood number." The shear-rate is considered more influential in vivo than the convection for small particles.
If an in vitro dissolution method is too sensitive (i.e., over discriminating), it may increase the manufacturers risk without any relevant effect on patient risk. By design, this occurs more often than that in the case of nondiscriminating methods, which do not reveal existing biorelevant differences. James Polli (University of Maryland) emphasized the common opinion that an overage of surfactants in the dissolution medium would reduce the sensitivity of dissolution tests. Moreover, some developers may not pay attention to the fact that for compositionally proportional dosage forms of different strengths, the sink conditions may not be given in all cases. This might reduce the discriminating power for the same dissolution methods when applied to higher dosage strengths of the same product. Polli pointed out that besides the sophisticated dissolution methods used during the development phase, a second dissolution method might be needed for QC purposes. He defined the meaning of predicting in vivo performance as the relationship between in vitro dissolution and the result of in vivo absorption, which is a composite of in vivo dissolution and permeation. He also introduced the importance of budgets into the discussion, a topic that would have been expected from industry representatives more so than from an academician. In fact, in vivo predictive dissolution is thought to reduce time and costs in drug development, resulting in a significantly reduced total number of clinical studies required. To waive these biostudies and rely solely on in vitro results may risk a delayed time to market, due to the need to complete additional biostudies. This biopharmaceutic risk is reflected by the number of IVIVC studies submitted for NDA. In cases where it is known that in vivo dissolution is limiting the absorption rate, such as for ER-dosage forms, the in vitro and in vivo dissolution may be similar. The total amount of ER dosage forms was $86 \%$ of the combined NDA from 2009 to 2012 (9). Another $6 \%$ were IR/ER combination products, and only $8 \%$ represented the group of IR dosage forms. It is not known whether the attempt to establish an in vivoin vitro relationship (IVIVR) was made, or was made but unsuccessful. Polli pointed out that the existing system of correlation levels from $A$ to $C$, as proposed by FDA and USP, can be extended to a level $D$ as a rank-order correlation. On the other hand, he introduced levels AA and AAA, by nonlinear functional relationships, as being superior to the existing linear IVIVC. He referred to his research in cases where dissolution was not clearly the rate-limiting step. His algorithm is based on the assumption that in vitro and in vivo dissolution rates are similar. Using a one-compartment, open model with first-order elimination invasion kinetics, his model deals with incomplete absorption of the dose at a given dissolution rate.

A closer look at the dissolution process of immediaterelease allows its categorization in mainly two steps, the disintegration and the dissolution of liberated drug particles. For classical disintegrating immediate-release oral tablets, disintegration testing may suffice to ascertain 
appropriate in vivo performance. This requires a relationship between disintegration and dissolution to be established. The international conference on harmonization (ICH) Q 6A guideline (10) provides a decision tree on how to substitute dissolution testing by disintegration testing based on the BCS principle. In the case of liquid-filled capsules, it becomes obvious that the rupture of the shell may be the rate-limiting step. In this case, meaningful in vitro testing can be reduced to the classical disintegration test. Modifications may be recommended in the case of gelatin capsules with cross-linking. The cross-linking process, catalyzed by ubiquitous aldehydes and ketones, slowly propagates during storage and leads to major changes in the in vitro dissolution behavior. Shells of aged capsules hydrolyze slower in comparison to freshly manufactured capsules, in particular at elevated $\mathrm{pH}$ values. This may be considered an artifact of the in vitro method because under in vivo conditions, proteases in the human $\mathrm{Gl}$ tract cleave the cross-linking bonds. Currently, USP is revising its General Chapter $<711>$ regarding the use of alternative proteolytic enzymes in dissolution media (11).

In USP General Chapter <2040> Disintegration and Dissolution of Dietary Supplements, two apparatus for disintegration testing are described, as Raimar Löbenberg (University of Alberta, Canada) explained. His work includes a comparison of the procedures for the disintegration and rupture test, with consideration to variations with the instruments involved (12). He elaborated that the specifications for the instruments involved with $\mathrm{ICH}$ allow great variability in the results due to variations in the hydrodynamic flow patterns, and the use of disks adds further variability. In his example, he showed statistically significant differences, without and with the use of disks, in the $95 \%$ confidence intervals of the means for the disintegration times. According to his experience, the common use of different media in dissolution testing may also apply to disintegration testing. He detected direct food-drug interactions with his in vitro approach. Using the GastroPlus software package for computer modeling, his working group in Alberta established IVIVC for selected drug products with an alternative dissolution approach (13).

\section{ORAL BIOPHARMACEUTICS TOOLS (ORBITO)}

Bertil Abrahamsson (AstraZeneca, Sweden) discussed the project "OrBiTo" from the European Innovative Medicines Initiative. Under his leadership, OrBiTo bundles the activities of 12 pharmaceutical companies, 11 universities, and other participants (14).

\section{OrBiTo Participants}

European Federation of Pharmaceutical Industries and Associations

- AstraZeneca AB, Sodertalje, Sweden

- AbbVie Deutschland GmbH \& Co. KG, WiesbadenDelkenheim, Germany
- Bayer Pharma AG, Berlin, Germany

- Boehringer Ingelheim International $\mathrm{GmbH}$, Ingelheim, Germany

- GlaxoSmithKline Research and Development Ltd, Brentford, United Kingdom

- H. Lundbeck A/S, Valby, Denmark

- Janssen Pharmaceutica NV, Beerse, Belgium

- Merck Sharp \& Dohme Corp, Rahway, NJ, United States

- Novartis Pharma AG, Basel, Switzerland

- Orion Corporation, Espoo, Finland

- Pfizer Ltd, Sandwich, United Kingdom

- Sanofi-Aventis Research and Development, ChillyMazarin, France

Universities, Research Organizations, Public Bodies, NonProfit Groups

- Uppsala Universitet, Uppsala, Sweden

- Copenhagen University, Copenhagen, Denmark

- Ernst Moritz Arndt University Greifswald, Greifswald, Germany

- Johann Wolfgang Goethe-Universität Frankfurt am Main, Frankfurt am Main, Germany

- Johannes Gutenberg Universität Mainz, Mainz, Germany

- Katholieke Universiteit Leuven, Leuven, Belgium

- Medical Products Agency, Uppsala, Sweden

- National and Kapodistrian University of Athens, Athens, Greece

- Netherlands Organization for Applied Scientific Research TNO, Delft, Netherlands

- University of Manchester, Manchester, United Kingdom

- University of Strathclyde, Glasgow, United Kingdom

Subject Matter Experts

- Simcyp Limited, Sheffield, United Kingdom

- Sirius Analytical Ltd, Forest Row, United Kingdom

- Simulations Plus, Inc., Lancaster, CA, United States

The goal of this project is to enhance the knowledge of how orally administered drugs are absorbed from the Gl tract and to create new lab tests and computer models that will better predict the performance of these drugs in patients.

Abrahamsson advertised the use of in vitro performance testing: "In vitro dissolution is a waste of time if not in vivo predictive!" This is a valid concern in development, and to a certain extent in QC testing as well, if a product-specific IVIVC is available. He summarized that progress was made in understanding $\mathrm{Gl}$ dissolution for controlled-release systems. Besides the determination of the "average" dissolution rate, these dosage forms require in vitro robustness tests of their rate-controlling mechanisms. Mechanical stress is one of the parameters that should be part of any meaningful IPD test.

Anette Müllertz (University of Copenhagen, Denmark) pointed out that physiological parameters such as the fasted $\mathrm{pH}$ (stomach $\mathrm{pH} 1.8-3$, duodenum $\mathrm{pH}$ 6-7, jejunum around $\mathrm{pH}$ 7.4) are known, as is the buffer capacity of Gl fluids. In the fasted state, osmolarity (stomach and duodenum around $200 \mathrm{mOsm} / \mathrm{L}$, jejunum $285 \mathrm{mOsm} / \mathrm{L}$ ), 
surface tension, bile salt, and lipid concentration for fluids in the proximal GI tract are available (15). The lipase units are not harmonized globally.

Higher variability of the intralumenal contents is expected for the postprandial state compared with the fasted state. The drug can encounter very different dissolution conditions within one subject and from subject to subject. Viscosity of intralumenal contents is significantly different from that of water. Consequently, in vivo dissolution is expected to be different if compared with the in vitro experiment. In the fed state, the amount and composition of food add further variability to the intestinal Gl fluids for which secretion rate and amount depend on the composition of food. For in vitro methods, there is no single medium that generally covers the "chemical space" of the fluid composition in the Gl tract. Therefore, Müllertz suggested the use of several different media. A part of her contribution to the OrBiTo project was to characterize the solubilizing properties of different potential dissolution media for selected drug substances such as aprepitant, carvedilol, felodipine, fenofibrate, probucol, and zafirlukast.

Disintegration testing was part of the standard QC methods for decades prior to dissolution testing. Disintegration testing was required for oral solid dosage forms at a time when tablet monographs were not yet in place. It has been acknowledged that proper drug release does not always require disintegration of the drug product prior to dissolution. However, for IR dosage forms, disintegration testing may be used in lieu of dissolution testing in case a relationship is established. This is already internationally harmonized and documented in the $\mathrm{ICH}$ Q6A document under Decision Tree \# 7 (10). Research was undertaken recently by Peter Langguth (University of Mainz, Germany) with the goal to adjust the classical in vitro disintegration test to the in vivo conditions. Media of different compositions and viscosities were used. One finding was that media with higher viscosity decelerate the disintegration process, which might be helpful for interpretation of food effects, though water diffusivity and film precipitation on the dosage form surface may hinder its wettability. Other factors that may have an impact on the initial water uptake as a prerequisite for disintegration are $\mathrm{pH}$, buffer strength, and surfactants.

Werner Weitschies' research (University of Greifswald, Germany) includes the use of magnetic resonance imaging (MRI) to study fluids and transport phenomena of drug products in the human GI tract. He pointed out that the fluid volumes in the stomach and small intestine were observed to be lower than that described over last decade due to improvement in investigational techniques. For instance, in the year 2005 the mean stomach volume was determined to be $105 \mathrm{~mL}$, whereas in 2014, with a significantly greater measuring precision, volume was reported to be $31 \mathrm{~mL}$. He could also demonstrate that under fed conditions, $240 \mathrm{~mL}$ of water given $30 \mathrm{~min}$ after the start of the study left the stomach following first-order kinetics, bypassing the food in the stomach. He called this phenomenon "Magenstrasse," which may be translated as "stomachroad," because concomitantly present food is emptied from the stomach relatively slowly by zero-order kinetics as a function of its caloric content. These differences in transit kinetics explain why the gastric emptying of dosage forms may also be influenced, depending on whether they are included in the rather solid food agglomerates or dispersed in the surrounding liquids. Compared with the highly variable gastric emptying, these studies describe a rather constant mean intestinal transit time between 3.5 and $4 \mathrm{~h}$. Weitschies argues that this "reliable" intestinal transit relates largely to the common study design with food intake after approximately the same time span, which then triggers the propagation of intestinal content. This is one example of the so-called common assumptions in the field of mechanistic prediction of in vivo performance.

Hans Lennernäs (Uppsala University, Sweden) introduced the regional human intestinal permeability $\left(P_{\text {eff }}\right)$, which is calculated from the disappearance rate of substances during intestinal perfusion. It is the most relevant variable in the prediction of absorption of drug compounds. One of the most important outcomes of his part in the OrBiTo project is a list of all historical $P_{\text {eff }}$ data obtained from 61 clinical studies performed for 80 substances. According to this report, a large database of $P_{\text {eff }}$ data for the proximal human small intestine is available, while a significant shortage of data from the distal small intestine and colon exists. The latter data are a prerequisite for accurate prediction of substances with low $P_{\text {eff }}$ and modified-release dosage forms. New experimental designs to close the knowledge gap are performed with a prompt regional intestinal release of drugs and successive blood sampling. As an internal standard for deconvolution purposes, an intravenous reference dose is applied.

Christel Bergström (Uppsala University, Sweden) investigated the molecular properties of drug substances that are relevant to dissolution. In her opinion, the BCS is not used to its full potential. From computational and experimental analyses of BCS Class 2 drug substances, it was concluded that by the current BCS classification, the acidic drug molecules may be deemed to have solubilitylimited absorption although complete absorption is found in vivo. Furthermore, lipids present in the intestine may increase the solubilization of compounds in the intestinal fluid, and compounds having log $D_{\mathrm{pH} 6.5} \geq 3.0$ were more soluble in fasted-state simulated intestinal fluid (FaSSIF). Bergström concluded that a more relevant $\mathrm{pH}$ restriction for acids (i.e., assessing the solubility solely at the $\mathrm{pH}$ of the small intestinal fluid) or physiologically relevant medium with lipids present such as FaSSIF should be used for the in vitro assessment of solubility and dissolution testing to better forecast solubility-limited absorption in vivo.

\section{ACADEMIA-REST OF WORLD}

At the University of Nottingham, United Kingdom, 
Luca Marciani's research group performed a study on the intestinal volume and the distribution of water, continuing the tradition of scientific research established in Nottingham for more than 25 years. In the past, many results at the University were obtained there by gamma scintigraphy. Currently, Marciani reported about the use of MRI snaphots in research. In fasted humans, the intestinal water is concentrated in small pockets. These discontinuous pockets were built after the ingestion of the standard $240 \mathrm{~mL}$ of water, as for a typical BA/BE study. The gastric water volume declined rapidly, within 12 min, releasing an average $94 \mathrm{~mL}$ of water into the small intestine, leading to approximately 15 pockets of $6 \mathrm{~mL}$ each. For the design of IPD, the small volume may be one important fact to consider, while the other factor is the turnover rate of fluids throughout $24 \mathrm{~h}$. The summarized volume turnover reaches about $10 \mathrm{~L}$, with only about $0.25 \mathrm{~L}$ not reabsorbed from the stool $(16,17)$.

The relationship between the rates of absorption and dissolution in vivo are the key for predictability of in vitro testing. Arik Dahan (University of Negev, Israel) cited Tsume and Amidon (18) with the statement that the higher the permeability, the more lax a dissolution criterion for granting a biowaiver may be. To explain unexpected findings from BA studies, variability in the permeability may vary throughout the Gl tract for many drug substances. Modeling may include this fact as well as the fact that pathophysiology can add further variability. Additionally, the variability in absorption is not restricted to the comparison of the small intestine as a whole to the colon, but may be found also within the three anatomical segments of the intestine: duodenum, jejunum, and ileum. This absorption rate discontinuity is accompanied by a gradient in $\mathrm{pH}$ value throughout the intestine. This is of importance to the in vivo dissolution of ionizable drugs. If absorption is not mainly driven by passive diffusion throughout the gut wall, carriers may then determine the rate of absorption. The distribution of certain carriers along the intestine may also be asymmetrical. In conclusion, the relationship of in vivo dissolution and absorption rates may flip along the GI tract more than once. With regard to the $\mathrm{Gl}$ transit rate, the predictability of in vivo performance may become rather difficult. Therefore, modeling should include different dissolution rates as a function of intralumenal $\mathrm{pH}$. It should also include variations in the times for the drug to reach those segments of different permeability.

IPD needs to define the in vivo measure or "validation" of the in vitro model. One of the leading discussion topics of the conference focused on whether the in vivo dissolution or rather the in vivo performance expressed as BA would be preferred. The bioavailability of drug product may be defined as the total output of partly overlapping processes. Oral drug absorption involves many processes that occur in parallel or sequential order, starting from the disintegration and dissolution of drugs in the Gl fluid, transit of the drug through the Gl tract, permeation of drug molecules across the intestinal membrane, and metabolism in the intestine or liver. Shinji Yamashita's research (Setsunan University, Japan) deals with water volume and transit rate of drugs in the Gl tract. His preferred method, which does allow monitoring the disintegration of dosage forms, is positron emission tomography. This technique requires a positron emitting radionuclide such as ${ }^{11} \mathrm{C}$ or ${ }^{18} \mathrm{~F}$. Either the drug substance or excipients, such as PEG, can be marked to allow monitoring of $\mathrm{Gl}$ transit or rupture of soft gelatin capsules in the proximal Gl tract. With this technique, the research group of Shinji Yamashita detected pharmacokinetic phenomena (e.g., the enterohepatic circulation for telmisartan). Interpretation of dissolution data for a drug product requires the knowledge of the physicochemical characteristics of the drug substance. Similar interpretation of BA data requires proper knowledge of the API pharmacokinetic behavior. This topic was one point of discussion, where it was clearly shown for carbamazepine that $60 \%$ of label claim dissolved in vitro would not allow for proper prediction of in vivo performance.

Kiyohiko Sugano (Toho University, Japan) provided a deep insight into the two basic equations that are key to the BCS, namely the Noyes-Whitney and the general permeability equation. He suggested extending the algorithms to the particle properties for ideally spherical particles first. Moreover, he expressed mathematically the influence of surfactants and implemented hydrodynamic parameters. Hydrodynamics has an influence on the thickness of the diffusion boundary layer. The diffusion rate may be a function of the Sharwood number and the Reynolds number.

The pivotal question asked by Marival Bermejo (University of Elche-Alicante) is, "How well can QC in vitro dissolution testing predict bioequivalence?" In an ideal situation, the dissolution test method can discriminate between product changes that affect product performance. The proper answer, however, requires an established IVIVC or at least an IVIVR. The approach for IR dosage forms is to integrate primarily a BCS categorization of the drug substance into the development process. Part of Bermejo's research was published recently $(19,20)$. In both of these publications, the reported probability of obtaining BE of drug products with similar dissolution profiles obtained with compendial dissolution methods was around $90 \%$ for BCS Class I and III drugs, expressed as post-test positive $\mathrm{BE}$ predictive value. However, the probability of false positive results for Class II drugs (i.e., non-BE but similar dissolution profiles) was computed to reach approximately $90 \%$. This may be taken as a clear indicator of a lack of predictive power of the compendial test methods. As an implementation of the BCS, the use of compendial instruments and pharmacopeial buffers covering the physiological $\mathrm{pH}$ range would improve the predictability of in vitro findings, preferably if the interpretation is done in combination with Caco-2 cell-based permeability study results. It is expected that this concept proposed by 
Bermejo is not only of certain relevance in the European Union but would extend to South America in particular if synchronized with the scientific concept applied by the World Health Organization.

\section{HOW IPD IS IMLPEMENTED IN INDUSTRY}

David Sperry (Eli Lilly and Company, Indianapolis, IN) described the use of a two-chamber dissolution instrument as the artificial stomach duodenum model (ASD), which captures supersaturation, precipitation, and dissolution phenomena as they occur in vivo to mimic the proximal Gl tract (stomach and duodenum). According to the decision tree presented by Sperry, ASD is applicable for nonconventional formulations or specifically for BCS Class Ilb or IVb drugs. ASD captures dynamic phenomena such as supersaturation, precipitation, and re-dissolution for BCS Class II and IV drugs, and it may be integrated in the method development process following a clearly defined decision tree to:

- Estimate in vivo solubility.

- Estimate drug substance dispersability.

- Determine the propensity for drug substance precipitation.

- Optimize formulations.

- Design QC test conditions.

- Assess the risk of changes observed with QC test conditions.

- Assess bioavailability risk.

- Input dissolution rates for absorption modeling. According to Michael Hawley (Boehringer Ingelheim, Ridgebury, (T), product development is based on three major features: in vivo performance, stability, and manufacturability. The least known feature is the in vivo performance, in cases when the in vitro dissolution results are not predictive. The in vivo corollary to in vitro testing is the pharmacokinetic profile obtained after administration of the product to healthy volunteers. The goal would be to have available dissolution methods that are, per se, biorelevant. Currently, none of the compendial instruments or dissolution media allow absolute prediction. Rather complex models are needed to link the in vivo and in vitro results on a product-by-product basis. Like the three pillars on which product design is based, the three aspects of a dissolution test including its operating conditions, dissolution media, and biorelevant specifications are linked to the product. In addition, if one of those is altered, the relationship will need to be reestablished. Release specifications may not apply throughout the shelf life of a product, but stability effects on the dissolution rate have to be evaluated. In some particular cases, a rather holistic dissolution test may detect relevant product changes that are not detectable by other techniques. Such was the case when during storage, an excipient interacted with the neutral salt of the API, prasugrel $\mathrm{HCl}$, partially converting the API into its free base. The $\mathrm{pH}$ of the dissolution medium was the pivotal characteristic to turn the QC dissolution method into one with in vivo predictive properties, due to the solubilities of both the unchanged parent compound and its stability compound. One of the conclusions drawn from the results generated by Boehringer-Ingelheim was to integrate the ASD model into the dissolution method and dosage form development prior to recommending a final QC test method.

One way of accelerating dissolution method development is to integrate computer simulations based on general assumptions such as those of the underlying BCS. Gordon Amidon's former coworker, John Crison (Bristol-Meyers Squibb), demonstrated actual industry practice on how to proceed. In cases of immediaterelease drug products with no pronounced impact of the dosage form properties, a second way uses mechanistic approaches based on the Nernst-Brunner equation to elaborate the impact of dissolution method variables on the dissolution rate of a drug with known physicochemical and pharmacokinetic properties. Fitting of mathematical models, such as the Weibull function, to the experimental data is a common approach and is provided by commercial software packages. A third way is to use statistical theories to link in vitro and in vivo findings, with the advantage that no particular pharmacokinetic model would be required. From a bird's eye view, it may turn out that the relationship of the Gl transit rate, dissolution rate, and absorption rate in the different segments of the GI tract are the BA-determining parameters of the particular dosage form under investigation, and by neglecting any leveling impact of the dosage form, solubility differences can be subsequently modeled. The composition of fluids and the physicochemical properties of concomitantly applied food can be of great interest, in particular for BCS Class II and IV drug substances. The alteration of gastric emptying by food adds further variability to the model. This fact requires solubility and hence dissolution experiments at different $\mathrm{pH}$ values and in the presence of surfactants as a reliable basis for computer modeling. For his development work, Crison uses software packages such as GastroPlus or SimCyp. For certain in vivo phenomena like $\mathrm{pH}$ changes, precipitation, or particle size distribution along the Gl tract, the computer models become more complex.

Ping Gao (AbbVie Inc., Chicago, IL) described how certain novel techniques are already used in his industrial research for in vivo predictive performance testing. He focused on understanding the supersaturated state of weak acid BCS Class II drugs in the Gl tract by determining the degree and duration of the supersaturation. Additionally, he used the in situ Focused Beam Reflectance Measurement (FBRM) to determine the onset of precipitation and the particle size distribution-time profile. FBRM allows the real-time in situ measurement of both particle size and the number of precipitated solid particles in test media and provides insight in the dominating process mechanisms such as nucleation, growth, and agglomeration (21). He concluded that certain formulations might cause 
supersaturation phenomena in the human $\mathrm{Gl}$ tract, which would improve the BA of selected poorly watersoluble drugs. To develop this type of formulation, some major considerations have to be made. Adequate dissolution is needed to achieve the supersaturation state. Subsequently, the duration and degree of supersaturation must be known. These may be influenced by the addition of polymeric crystallization inhibitors. Such dosage-form technology would require a biphasic dissolution test to characterize dosage form performance. The biphasic system allows simulation of the absorption process in a particular way. It is, in fact, a two-compartment dissolution model, which goes in line with the compartmental models used in pharmacokinetics.

The goal of Quality by Design in pharmaceutical development is to design a quality product and its manufacturing process and to consistently deliver the intended performance of the product. At a minimum, those aspects of drug substances, excipients, container closure systems, and manufacturing processes that are critical to product quality should be determined, and control strategies should be justified according to ICH Q8(R2) (22). Jack Cook (Pfizer Inc., Chicago, IL) reported about strategies in his personal industrial work environment. He noted that IVIVC studies are often conducted too late in development (i.e., phase 2 or 3), and he instead suggested attempting IVIVC in earlier phases, since changes in formulation and manufacturing during development are opportunities to gain additional knowledge, further supporting the establishment of the design space (23). He expanded his expectations from the classical BCS to the subclasses of the BCS to increase the likelihood of prediction. In summary, Classes I and III provide a safe zone. Class Ila may allow establishment of a Level A IVIVC, whereas Class IIB may be challenging due to precipitation. All three subclasses for BCS Class IV could provide the opportunity for a case-bycase IVIVC.

To speed up development, Cook introduced the concept of a "Synthetic Solution." The data may be taken from the literature, as he explained for the example of a study performed on ER metoprolol and an oral solution. This allowed the creation of a solution profile as a unit impulse. With additional steps, this allowed the computation of apparent in vivo absorption kinetics for different dosage forms. The subsequent comparison of in vitro and in vivo profiles may help to improve the in vitro method already at an early stage or may indicate that the in vivo assumptions are erroneous (e.g., absorption windows may exist). Cook emphasized the importance of early IVIVC attempts when changes in the formulation are the largest, as mentioned above. Moreover, the development of a predictive dissolution test should also be undertaken in early formulation development.

\section{CONTRACT RESEARCH ORANIZATIONS SPECIALIZED IN IPD}

Dissolution is a testing methodology for dosage forms, but often drug substance properties may be masked by the technology of the dosage form. Biorelevant in vitro testing may be close to in vivo conditions in the case of IR dosage forms. For ER dosage forms, it may reflect the rate and amount by which the active moiety is absorbed from the dosage form, that is, its bioavailability (24). According to Johannes Krämer (PHAST GmbH, Germany), biorelevance may have different meanings depending on whether the API is for local or systemic application and whether the drug release follows a stochastic process or is governed by a rate-controlling mechanism. The volume and composition of media in vivo are variable. The in vitro media are preferably simplified but still reflect the pivotal properties of the in vivo solubilizing characteristics. The volume and composition of in vitro dissolution media depend on the experimental design of the test. If properly developed, compendial instruments and simple buffer systems may be predictive of in vivo product performance. The use of biorelevant media, as first described by Dressman et al. (25), may be beneficial in preclinical and development phases. Biorelevant dissolution testing is a topic of other scientific organizations as well. A report about the progress is available for a recent AAPS/FIP workshop in Bethesda, MD (26).

Compendial dissolution testing is linked to manufacturing, but Dwayne Friesen (Bend Research Inc., Bend, OR) criticized that those methods often would not allow the measurement of critical quality attributes of drug products. Therefore, the testing does not predict the in vivo performance of the drug product. In the preclinical and early development series of dissolution testing, methods are used to quantify a formulation property under in vivo conditions. Results are then combined to build a mechanistic model.

Still a challenge to mimic by dissolution testing are:

- Complexity of human Gl tract.

- Inter- and intrasubject variability.

- Variability in transit time as a function of food and dosage form.

- Dynamic nature of excretion and absorption in the Gl tract.

Computational modeling sometimes uses software originally developed for simulation of chemical processes, and further development is required to better fit the goals of establishing an IVIVC. 


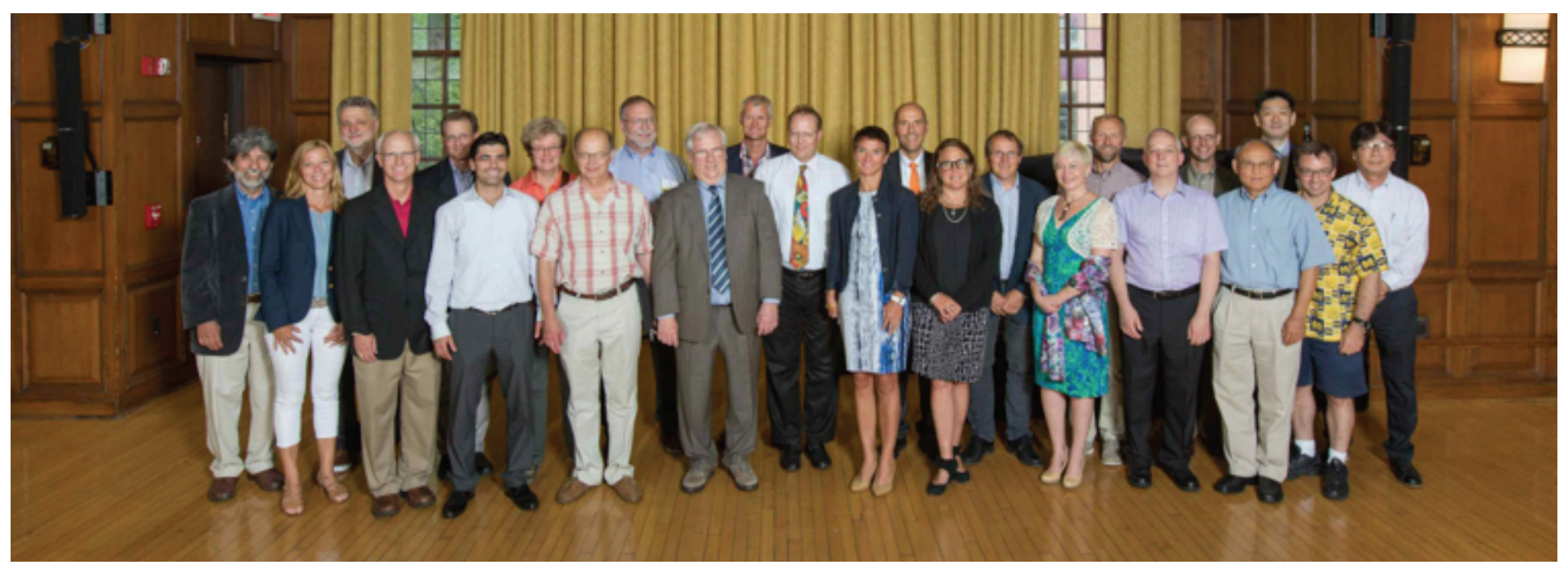

Speakers from left to right: James Brasseur, Christel Bergström, Peter Langguth, Gregory Amidon, Michael Hawley, Arik Dahan, Nair Rodriguez-Hornedo, Dwayne Friesen, James Cook, Gordon Amidon, Hans Lennernäs, Raimar Löbenberg, Erika Stippler, Johannes Krämer, Anette Müllertz, Werner Weitschies, Marival Bermejo, Bertil Abrahamsson, Luca Marciani, David Sperry, Ping Gao, Kiyohiko Sugano, James Polli, Shinji Yamashita.

\section{REFERENCES}

1. Amidon, G. L. Mol. Pharmaceutics, in press.

2. Skwierczynski, R.; Curry, P.; Gray, V.; Krämer, J.; Stippler, E.; Suggett, J.; Mirza, T.; Brown, W. Stimuli to the revision process: Revision of The Dissolution Procedure: Development and Validation <1092>. Pharm. Forum 2014, 40 (1). http://www.usppf.com/pf/pub/index. html.

3. FY 14 GDUFA Regulatory Research Priorities. U.S. Food and Drug Administration. http://www.fda.gov/drugs/ newsevents/ucm367997.htm (accessed Feb 16, 2015).

4. FY 2013 Awarded GDUFA Regulatory Research Contracts and Grants. Food and Drug Administration. http://www.fda.gov/downloads/Forlndustry/ UserFees/GenericDrugUserFees/UCM386955.pdf (accessed Feb 16, 2015).

5. Rhie, J. K.; Hayashi, Y.; Welage, L. S.; Frens, J.; Wald, R. J.; Barnett, J. L.; Amidon, G. E.;Putcha, L.; Amidon, G. L. Drug Marker Absorption in Relation to Pellet Size, Gastric Motility and Viscous Meals in Humans. Pharm. Res. 1998, 15 (2), 233-238. DOI: 10.1023/A:1011962501270.

6. Tsume, Y.; Mudie, D. M.; Langguth, P.; Amidon, G. E.; Amidon, G. L. The Biopharmaceutics Classification System: Subclass for in vivo predictive dissolution (IPD) methodology and IVIVC. Eur. J. Pharm. Sci. 2014, 57, 152-163. DOI: 10.1016/J.EJPS.2014.01.009.

7. Lennernäs, H.; Ahrenstedt, O.; Hällgren, R.; Knutson, L.; Ryde, M.; Paalzow, L. K. Regional Jejunal Perfusion, a New in Vivo Approach to Study Oral Drug Absorption in Man. Pharm. Res. 1992, 9 (10), 1243-1251. DOI: 10.1023/A:1015888813741.

8. Mudie, D. M.; Amidon, G. L.; Amidon, G. E. Physiological
Parameters for Oral Delivery and in Vitro Testing. Mol. Pharmceutics 2010, 7 (5), 1388-1405. DOI: 10.1021/ mp100149j.

9. Suarez Sharp, S. FDA's Experience on IVIVC-New Drug Products. Presented at PQRI Workshop on Application of IVIVC in Formulation Development, Bethesda, MD, September 5-6, 2012.

10. International Conference on Harmonisation ofTechnical Requirements for Registration of Pharmaceuticals for Human Use. Specifications: Test Procedures and Acceptance Criteria for New Drug Substances and New Drug Products: Chemical Substances, Q6A; ICH Harmonised Tripartite Guideline: Geneva, Switzerland, 1999. http://www.ich.org/fileadmin/Public_Web_ Site/ICH_Products/Guidelines/Quality/Q6A/Step4/ Q6Astep4.pdf (accessed Feb. 16, 2015).

11. Barker, A. R.; Krämer, J. Summary Report from the USP Workshop on Dissolution Testing of Capsules. Dissolution Technol. 2014, 21 (4), 67-69. DOI: 10.14227/ DT210414P67.

12. Donauer, N.; Löbenberg, R. A mini review of scientific and pharmacopeial requirements for the disintegration test. Int. J. Pharm. 2007, 345 (1-2), 2-8. DOI: 10.1016/j. ijpharm.2007.08.045.

13. Okumu, A.; DiMaso, M.; Löbenberg, R. Dynamic Dissolution Testing To Establish In Vitro/In Vivo Correlations for Montelukast Sodium, a Poorly Soluble Drug. Pharm. Res. 2008, 25 (12), 2778-2785. DOl: 10.1007/s11095-008-9642-z.

14. OrBiTo, Oral biopharmaceutics tools. Innovative Medicines Initiative. http://www.imi.europa.eu/ content/orbito (accessed Feb 16, 2015). 
15. Pedersen, P. B.; Vilmann, P.; Bar-Shalom, D.; Müllertz, A.; Baldursdottir, S. Characterization of fasted human gastric fluid for relevant rheological parameters and gastric lipase activities. Eur. J. Pharm. Biopharm. 2013, 85 (3), 958-965. DOI: 10.1016/j.ejpb.2013.05.007.

16. Thelen, K.; Coboeken, K.; Willmann, S.; Burghaus, R.; Dressman, J. B.; Lippert, J. Evolution of a detailed physiological model to simulate the gastrointestinal transit and absorption process in humans, Part 1: Oral solutions. J. Pharm. Sci. 2011, 100 (12), 5324-5345. DOI: 10.1002/jps.22726.

17. Spiller, R. C. Intestinal absorptive function. Gut 1994, 35, S5-S9. DOI: 10.1136/gut.35.1_Suppl.S5.

18. Tsume, Y.; Amidon, G. L. The Biowaiver Extension for BCS Class III drugs: The Effect of Dissolution Rate on the Bioequivalence of BCS Class III ImmediateRelease Drugs Predicted by Computer Simulation. Mol. Pharmaceutics 2010, 7 (4), 1235-1243. DOI: 10.1021/ mp100053q.

19. Cristofoletti, R.; Chiann, C.; Dressman, J. B.; Storpirtis, S. A comparative analysis of biopharmaceutics classification system and biopharmaceutics drug disposition classification system: A cross-sectional survey with 500 bioequivalence studies. J. Pharm. Sci. 2013, 102 (9), 3136-3144. DOI: 10.1002/jps.23515.

20. Ramirez, E.; Laosa, O.; Guerra, P.; Duque, B.; Mosquera, B.; Borobia, A. M.; Lei, S. H.; Carcas, A. J.; Frias, J. Acceptability and characteristics of 124 human bioequivalence studies with active substances classified according to the Biopharmaceutic Classification System. Br. J. Clin. Pharmacol. 2010, 70 (5), 694-702. DOI: 10.1111/j.1365-2125.2010.03757.x.
21. Gao, P.; Akrami, A.; Alvarez, F.; Hu, J.; Li, L.; Ma, C.; Surapaneni, S. Characterization and optimization of AMG 517 supersaturatable self-emulsifying drug delivery system (S-SEDDS) for improved oral absorption. J. Pharm. Sci. 2009, 98 (2), 516-528. DOI: 10.1002/jps.21451.

22. International Conference on Harmonisation ofTechnical Requirements for Registration of Pharmaceuticals for Human Use. Pharmaceutical Development, Q8(R2); ICH Harmonised Tripartite Guideline: Geneva, Switzerland, 2009. http://www.ich.org/fileadmin/Public_Web_Site/ ICH_Products/Guidelines/Quality/Q8_R1/Step4/Q8_ R2_Guideline.pdf (accessed Feb 16, 2015).

23. Cook, J. A. Development strategies for IVIVC in an industrial environment. Biopharm. Drug Dispos. 2012, 33 (7), 349-353. DOI: 10.1002/bdd.1791.

24. Leeson, L. J.; Adair, D.; Clevenger, J.; Chiang, N. The In Vitro Development of Extended-Release Solid Oral Dosage Forms. J. Pharmacokinet. Biopharm. 1985, 13 (5), 493-514. DOI: 10.1007/BF01059332.

25. Dressman, J. B.; Amidon, G. L.; Reppas, C.; Shah, V. P. Dissolution Testing as a Prognostic Tool for Oral Drug Absorption: Immediate Release Dosage Forms. Pharm. Res.1998,15(1),11-22.DOI:10.1023/A:1011984216775.

26. Reppas, C.; Friedel, H.-D.; Barker, A. R.; Buhse, L. F.; Cecil, T. L.; Keitel, S.; Krämer, J.; Morris, J. M.; Shah, V. P.; Stickelmeyer, M. P.; Yomota, C.; Brown, C. K. Biorelevant In Vitro Performance Testing of Orally Administered Dosage Forms-Workshop Report. Pharm. Res. 2014, 31 (7), 1867-1876. DOI: 10.1007/s11095-014-1348-9. 